\title{
MHD Stagnation-Point Flow of Non-Newtonian Fluid and Heat Transfer over Stretching/Shrinking Sheet in a Porous Medium
}

\section{Ala'a Abdul-Raheem Hammodat Anas Ihsan Basheer Department of Mathematics College Education of Pure Science Mosul University}

\author{
Received \\ 25 / $01 / 2018$ \\ Accepted \\ 03 / 04 / 2018
}

\begin{abstract}
الخلاصة
في هذا البحث، قمنا بدراسة الجريان في نقطة الركود وانتقال الحرارة لمائع لا نيوتوني

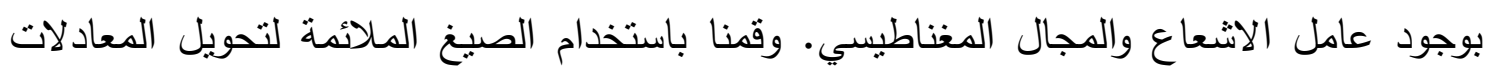

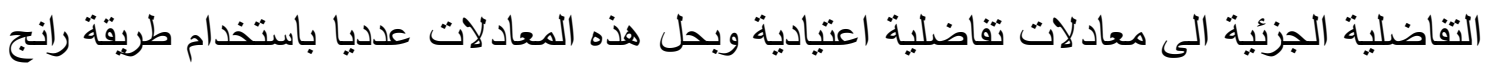

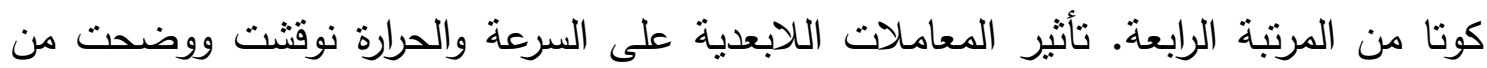

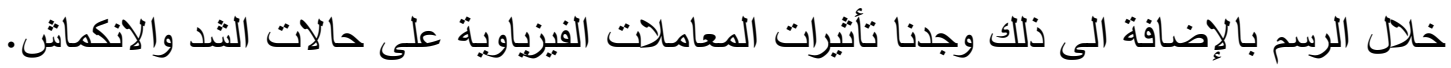

\begin{abstract}
In this work, we study the stagnation point flow of Casson fluid and heat transfer phenomena in presence of thermal radiation and magnetic field. We used the suitable similarity transformation to reduce governing partial differential equations into ordinary differential equations, these equations are solved numerically using Rang-Kutta method. Effects of nondimensional parameters on velocity, temperature are discussed and presented through graphs as well as coefficient of skin friction and local Nusselt numbers is investigated stretching/shrinking cases.
\end{abstract}

Keywords: Casson Fluid, Stagnation point flow, Heat transfer, Shrinking/stretching sheet, Skin Friction coefficient, Porous medium . 


\section{Introduction:}

In fluid dynamics the effects of external magnetic field on magnetohydrodynamic (MHD) flow over a stretching/shrinking sheet are very important due to its applications in many engineering problems, such as glass manufacturing, geophysics, paper production, and purification of crude oil [6].In 1936, Homann F. proposed the solution of two dimensional flow of fluid near stagnation point [2].In 1970, Crane L. J. studied the flow due to stretching of a flat surface [10]. In 1974, Pavlov K. B. discussed the effect of external magnetic field on the MHD flow of viscous fluid on the stretching sheet [8]. In 1992, Andersson H. I. reported the MHD flow of viscous fluid on a stretching sheet [3]. In 2005, Mukhopadhyay S. , Layek G. C. and Samad S. A. studied the MHD flow and heat transfer over a stretching sheet with variable fluid viscosity [14].In 2010, Fang T. , and Zhang J. proposed the exact solution of MHD flow due to shrinking sheet with wall mass suction [16]. At the same year, Bhattacharyya K., and Layek G.C. discussed the behavior of solute distribution in MHD boundary layer flow past a stretching sheet[7]. In 2011, Bhattacharyya K. showed the effects of radiation and heat source/sink on unsteady MHD boundary layer flow and heat transfer over a shrinking sheet with suction/injection, as well as we reported that the effects of heat source/sink on MHD flow and heat transfer over a shrinking sheet with mass suction [5]. In 2012, Salem A. M. , and Fathy R., studied the effects of variable properties on MHD heat and mass transfer flow near a stagnation point towards a stretching sheet in a porous medium with thermal radiation [1]. At the same year, Mukhopadhyay S. ,and Gorla R. S. R. proposed effects of partial slip on boundary layer flow past a permeable exponential stretching sheet in presence of thermal radiation [15]. In 2013, Bhattacharya K. , Vajravelu K. and Hayat T. , reported the slip effects on parametric space and solution for the boundary layer flow due to non-porous stretching and shrinking sheet [9]. At the same year, Bhattacharyya K. , studied the MHD stagnation point flow of Casson fluid and heat transfer over a stretching sheet with thermal radiation [6]. In 2015, Ahmed J., shahzad A., Khan M., and Ali R., takes a note on convective heat transfer of MHD Jeffrey fluid over a stretching sheet [4].In 2016, Mahantesh M. N. and Shilpa J. M. reported the stagnation point flow of non-Newtonian fluid and heat transfer over a stretching/shrinking sheet in a porous medium [12]. In the present paper, the steady two dimensional MHD stagnation point flow of non-Newtonian (Casson) fluid and heat transfer of a stretching /shrinking sheet in presence of thermal radiation are investigated. The governing partial differential equations are converted into the non-linear ordinary differential equations using suitable similarity transformations. These ordinary differential equations are solved numerically using Runge-Kutta fourth order method with efficient numerical shooting method. The numerical results are plotted 
in some figures to see the effects of physical parameters on the flow and heat transfer.

\section{Mathematical Formulation:}

Consider a steady stagnation point flow of incompressible flow of electrically conducting Casson fluid induced by stretching/shrinking sheet in a porous media. The cartesian co-ordinates $\mathrm{x}$ and $\mathrm{y}$ are taken with the origin $\mathrm{O}$ at the stagnation point, and are defined such that the $\mathrm{x}$-axis is measured along the stretching/shrinking sheet and the y-axis is measured normal to it. It is assumed that the velocity of the external flow is given $u_{e}(x)=a x$, where $a>0$ is the strength of the stagnation flow and the surface temperature $T_{w}$ is a constant. It is also assumed that the velocity of stretching/shrinking sheet is given by $u_{w}(x)=b x$, where $\mathrm{b}$ is the stretching rate, with $b>0$ and $b<0$ are for stretching/shrinking case respectively. The flow configuration is shown in Fig.1.

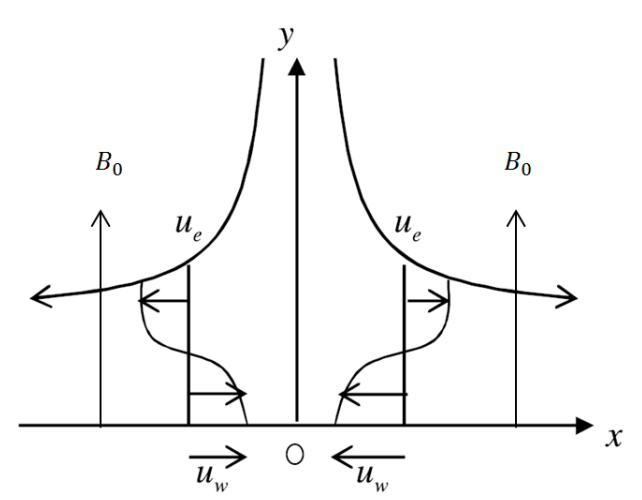

a. Shrinking case

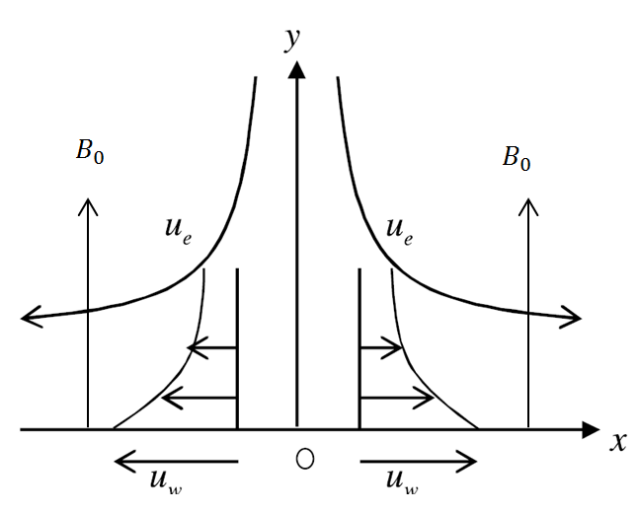

b. stretching case

Fig.(1) The geometry of Model ( a. shrinking case, b. stretching case )

The rheological equation of state for an isotropic and incompressible flow of a Casson fluid can be written as[11].

$$
\tau_{i j}= \begin{cases}2\left(k_{c}+\frac{\tau_{0}}{\sqrt{2 \pi}}\right) e_{i j}, & \pi>\pi_{c} \\ 2\left(k_{c}+\frac{\tau_{0}}{\sqrt{2 \pi_{c}}}\right) e_{i j} & , \pi<\pi_{c}\end{cases}
$$

Where

$$
e_{i j}=\frac{1}{2}\left(\frac{\partial u_{i}}{\partial x_{j}}+\frac{\partial u_{j}}{\partial x_{i}}\right)
$$


is the rate of strain tensor, $k_{c}$ is the Casson's coefficient of viscosity, $\pi$ is the product of the component of deformation rate with itself, $\pi_{c}$ is the critical value of the product of the component of the rate of strain tensor with itself, $\tau_{0}$ is the yield stress of the fluid and $u_{i}$ and $u_{j}$ are the velocity components.

Under these previous conditions, the MHD boundary layer equations for steady stagnation - point flow can be written as :

$$
\begin{aligned}
& \frac{\partial u}{\partial x}+\frac{\partial v}{\partial y}=0 \\
& u \frac{\partial u}{\partial x}+v \frac{\partial u}{\partial y}=u_{e} \frac{d u_{e}}{d x}+v\left(1+\frac{1}{\beta}\right) \frac{\partial^{2} u}{\partial y^{2}}+\left(1+\frac{1}{\beta}\right) \frac{v}{k}\left(u_{e}-u\right)+g \beta\left(T-T_{\infty}\right)-\ldots \\
& \ldots \ldots-\frac{\sigma B_{0}^{2}}{\rho} u
\end{aligned}
$$

Where $u$ and $v$ are the velocity component in $x$ and $y$ directions respectively, $v$ is kinematic fluid viscosity, $\rho$ is the fluid density, $\beta=k_{c} \sqrt{2 \pi_{c}} / \tau_{0}$ is the non-Newtonian or Casson parameter, $k$ is the permeability of the porous medium, $\sigma$ is the electrical conductivity of the fluid, and $B_{0}$ is the strength of magnetic field applied in the $y$ direction, with the induced magnetic field being neglected. For the temperature distribution in the flow field with the thermal radiation.

The governing energy equation can be written as:

$u \frac{\partial T}{\partial x}+v \frac{\partial T}{\partial y}=\alpha \frac{\partial^{2} T}{\partial y^{2}}-\frac{1}{\rho c_{p}} \frac{\partial q_{r}}{\partial y}$

Where $T$ is the temperature, $c_{p}$ is the specific heat and $q_{r}$ is the radiative heat flux. Using the Roseland approximation for radiation [13], $q_{r}=-\left(4 \sigma^{*} / 3 k_{1}\right) \frac{\partial T^{4}}{\partial y}$ is obtained, where $\sigma^{*}$ is the Stefan-Boltzmann constant and $k_{1}$ is the absorption coefficient, we presume that the temperature variation within the flow is such that $T^{4}$ may be expanded in a Taylors series. Expanding $T^{4}$ about $T_{\infty}$ and neglecting higher- order terms we get $T^{4}=4 T_{\infty}^{3} T-3 T_{\infty}^{4}[6]$.

Now equation (5) reduces to 
$u \frac{\partial T}{\partial x}+v \frac{\partial T}{\partial y}=\left[\alpha+\frac{16 \sigma T_{\infty}^{3}}{3 \rho c_{p} k_{1}}\right] \frac{\partial^{2} T}{\partial y^{2}}$

Where $\alpha=\frac{k}{\rho c_{p}}$ is thermal diffusivity.

The boundary conditions for the problem are :

$u_{w}(x)=b x \quad, \quad T=T_{w} \quad$ at $\quad y=0$

$u_{e}(x)=a x \quad, \quad T=T_{\infty} \quad$ at $\quad y \rightarrow \infty$

\section{Equations and Non-dimensional Form:}

In order to change the differential equations of the model to be more convenient to the mathematical model, we introduce the following similarity variables,

$\eta=\left(\frac{u_{e} x}{v}\right)^{1 / 2} \frac{y}{x} \quad, \quad \psi=\left(v x u_{e}\right)^{1 / 2} f(\eta) \quad, \quad \theta(\eta)=\frac{T-T_{\infty}}{T_{w}-T_{\infty}}$

From equations (7) and (8), we get :

$\eta=\sqrt{\frac{a}{v}} y, \quad \psi=\sqrt{a v} x f(\eta) \quad, \quad \theta(\eta)=\frac{T-T_{\infty}}{T_{w}-T_{\infty}}$

Where $\psi$ is the stream function defined as $u=\frac{\partial \psi}{\partial y}$ and $v=-\frac{\partial \psi}{\partial x}$ which identically satisfy equation (3) and substituting the above transformation equation (9) and equations (4) and (6) we obtain:

$$
\begin{aligned}
& \left(1+\frac{1}{\beta}\right)\left(f^{\prime \prime \prime}+K\left(1-f^{\prime}\right)\right)+f f^{\prime \prime}-f^{\prime^{2}}-M f^{\prime}+\lambda \theta+1=0 \\
& \theta^{\prime \prime}-R f \theta^{\prime}=0
\end{aligned}
$$

Where $K=\frac{v x}{u_{e} k_{1}} \quad$ is the porosity parameter of the porous medium .

$$
\begin{aligned}
& M=\frac{\sigma B_{0}^{2}}{a \rho} \text { is the magnetic parameter. } \\
& \lambda=\frac{g \beta T_{0}}{a^{2} x} \text { is the mixed convection parameter. } \\
& R=\frac{3 \rho c_{p} k_{1}}{3 k k_{1}+16 \sigma T_{\infty}^{3}} \text { is the thermal radiation parameter. }
\end{aligned}
$$


The boundary conditions reduce to :

$$
\begin{aligned}
& f(\eta)=0 \quad, \quad f^{\prime}(\eta)=\frac{b}{a}=c \quad, \theta(\eta)=0 \quad \text { at } \quad \eta=0 \\
& f^{\prime}(\eta) \rightarrow 1 \quad, \quad \theta(\eta) \rightarrow 0 \quad \text { at } \quad \eta \rightarrow \infty
\end{aligned}
$$

\section{The Physical Quantities:}

The important physical quantities are the wall skin friction coefficient $C_{f}$ and the local Nusselt number $N_{u_{x}}$ which can be defined as :

$$
C_{f}=\frac{\tau_{w}}{\rho u_{e}^{2}(x)} \quad \text { and } \quad N_{u_{x}}=\frac{x q_{w}}{k\left(T_{w}-T_{\infty}\right)}
$$

Where $\tau_{w}$ is the shear stress or skin friction along stretching sheet and $q_{w}$ is the heat flux from the sheet and those are defined as :

$\tau_{w}=\left(k_{c}+\frac{\tau_{0}}{\sqrt{2 \pi_{c}}}\right)\left(\frac{\partial u}{\partial y}\right)_{y=0} \quad$ and $\quad q_{w}=-k\left(\frac{\partial T}{\partial y}\right)_{y=0}$

Thus, we get the skin friction coefficient $C_{f}$ and the local Nusselt number $N_{u_{x}}$ as follows :

$C_{f}\left(R_{e}\right)^{1 / 2}=f^{\prime \prime}(0) \quad$ and $\quad\left(R_{e}\right)^{-1 / 2} N_{u_{x}}=-\theta^{\prime}(0)$

Where $R_{e}=\frac{u_{e} x}{v}$ is the local Reynolds number .

\section{Numerical Method for Solution:}

Equations (10) and (11) along with boundary conditions (12) are solved using Rang-Kutta method [12] by converting them to an initial value problem. In this method it is necessary to choose a suitable finite value $\eta \rightarrow \infty$. The following system is set :

$y_{1}^{\prime}=f^{\prime} \quad, \quad y_{2}^{\prime}=f^{\prime \prime}$

$y_{3}^{\prime}=\frac{1}{\left(1+\frac{1}{\beta}\right)}\left[-y_{1} y_{3}+\left(K\left(1+\frac{1}{\beta}\right)+M\right) y_{2}-\lambda y_{5}-1-K\left(1+\frac{1}{\beta}\right)\right]$

$y_{5}^{\prime}=\theta^{\prime} \quad, \quad y_{6}^{\prime}=R y_{1} y_{6}$

With the boundary conditions 


$$
\begin{array}{llll}
y_{1}=0, & y_{2}=\frac{b}{a}=c \quad, \quad y_{5}=0 \quad \text { at } \quad \eta=0 \\
y_{2} \rightarrow 1, & y_{5} \rightarrow 0 \quad \text { at } \quad \eta \rightarrow \infty
\end{array}
$$

In order to integrate (16) and (17), the value for $f^{\prime \prime}(0)$ and $\theta^{\prime}(0)$ are required, but no such values are given at the boundary, the suitable guess value for $f^{\prime \prime}(0)$ and $\theta^{\prime}(0)$ are chosen and then integration is carried out, then the calculate values for $f^{\prime}$ and $\theta$ at $\eta_{\infty}$ are compared with the given boundary conditions $f^{\prime}(\infty)=1$ and $\theta(\infty)=0$ and the estimated values $f^{\prime \prime}(0)$ and $\theta^{\prime}(0)$ are adjusted to give a better approximation for the solution. The values for $f^{\prime \prime}(0)$ and $\theta^{\prime}(0)$ are taken and by using the Fourth order classical Rang- Kutta method the problem is solved. The above procedure is repeated until the asymptotically converged results within a tollerence level $10^{-5}$ are obtained and the step size chosen is $\Delta \eta=0.001$.

\section{Results and Discussions:}

The numerical computations have been carried out using above described Rank-Kutta method for several values of physical parameters such as Casson parameters $\beta$, porosity parameter $K$, magnetic field $M$ and radiation parameter $R$ as shown in graphs Fig.2-Fig.11. to study the velocity field Fig.2, Fig3.and Fig.4 shows the influence of the Casson parameter $\beta$, porosity parameter $K$ and magnetic field $M$ on the velocity profiles, we observe that the magnitude of velocity in the boundary layer increase with an increase in the Casson fluid parameter $\beta$. While the magnitude of velocity is decreases with an increases of porosity parameter and magnetic field respectively. Fig5 represent the effect of porosity parameter $K$ with the temperature profile, it is show that the temperature is decrease at the porosity parameter is increase.Fig6 and Fig.7 represents the variation of skin friction coefficient with parameter $c$ for $\beta=0.1,0.2,0.5$ and $K=0.5,1,1.5$.It is observe that with increases values of Casson parameter $\beta$ and porosity parameter $K$, the skin friction coefficient is decrease.Fig.8 represent the temperature profile gradient with $c$ for $R=2,4,5$, it is noticed that the temperature is increase when the radiation parameter $R$ is increase.Fig.9 and Fig.10 represents the variation of skin friction coefficient the temperature profile gradient with parameter $M$ for $\beta=0.1,0.2,0.5$ and $R=2,4,5$ respectively, it is observed that the skin friction coefficient is decrease when $\beta$ is increase the temperature is increase when the radiation parameter $R$ is increase. 


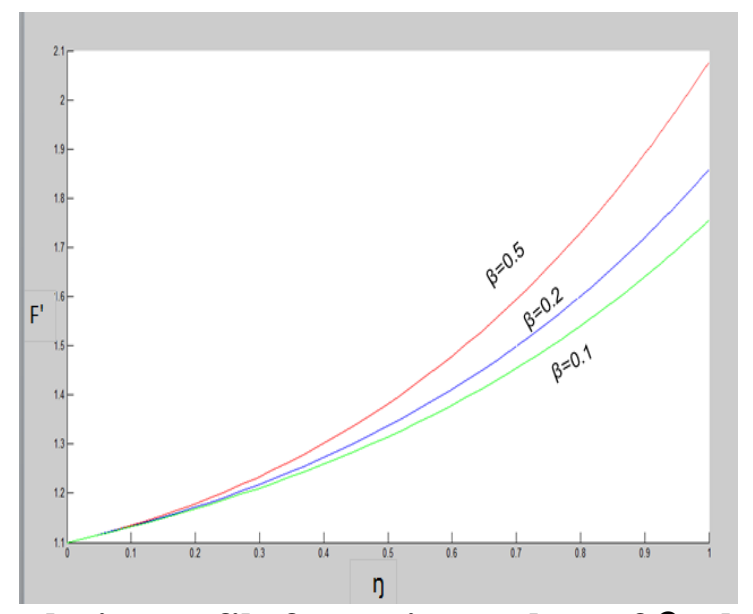

Fig.2. Variation of velocity profile foe various values of $\beta$ when $K=2, M=1, \lambda=1$

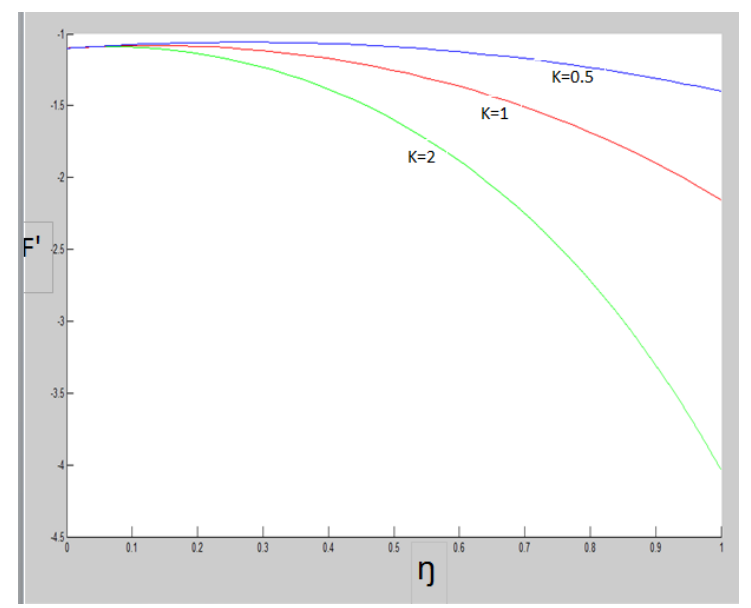

Fig.3. Variation of velocity profile foe various values of $\mathrm{K}$ when $\boldsymbol{\beta}=0.5, \mathrm{M}=1, \lambda=$ $1, R=5$

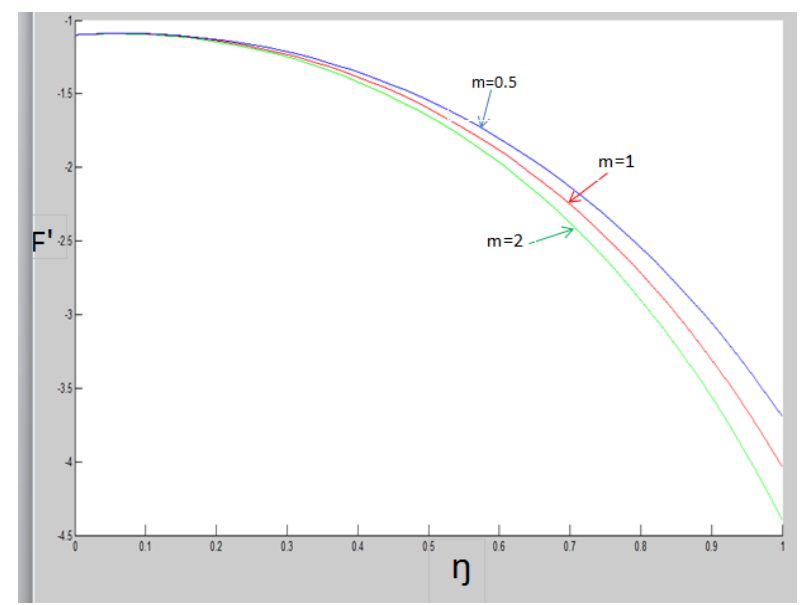

Fig.4. Variation of velocity profile foe various values of $M$ when $\beta=0.5, K=2$, $\lambda=1$, and $R=5$ 


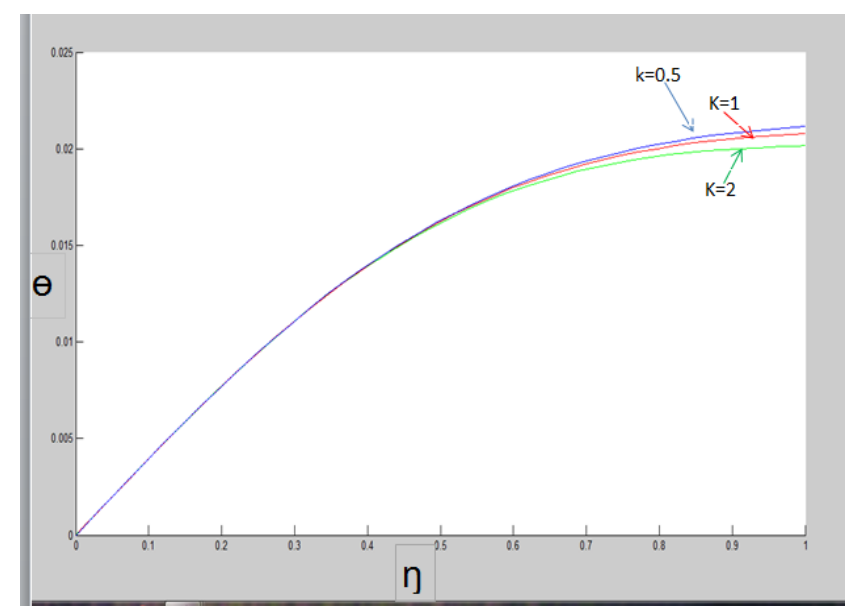

Fig.5. Variation of temperature profile foe various values of $\mathrm{K}$ when $\beta=0.5, \lambda=1$, $\mathrm{M}=\mathbf{1}, \mathrm{R}=\mathbf{5}$

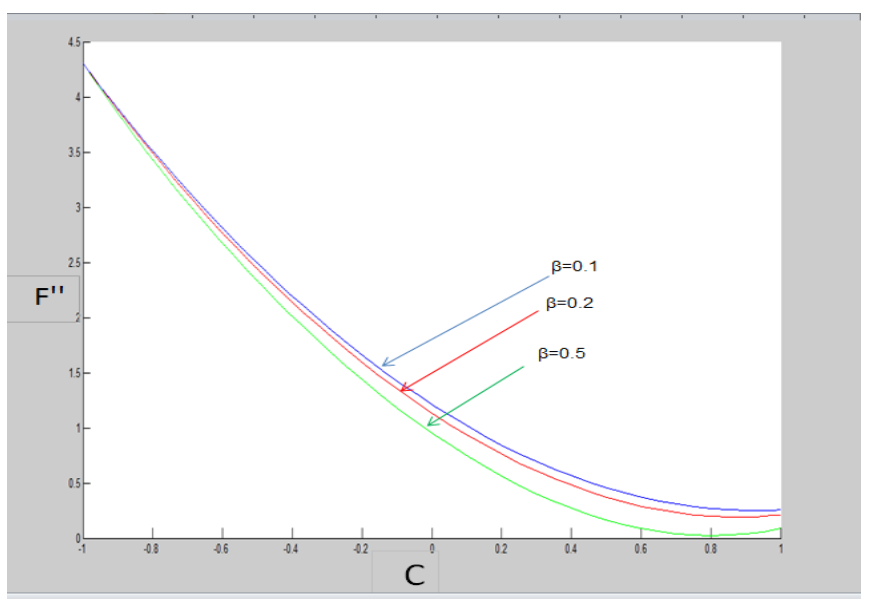

Fig.6. Variation of skin friction coefficient $f^{m}(0)$ with $C$ for various values of $\beta$ when $K=2, \lambda=1, M=1$

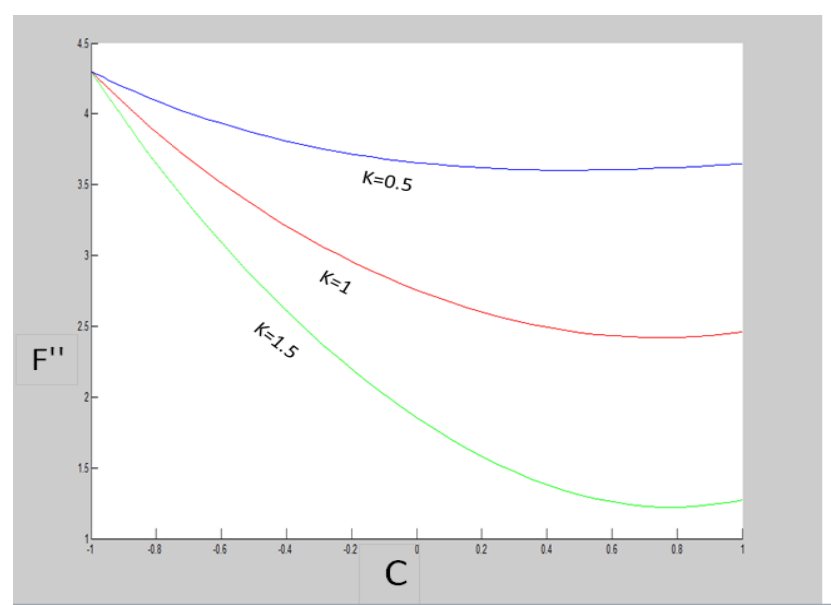

Fig.7. Variation of skin friction coefficient $f^{\prime \prime}(0)$ with $C$ for various values of $K$ when $\beta=0.5, \lambda=1, M=1$ 


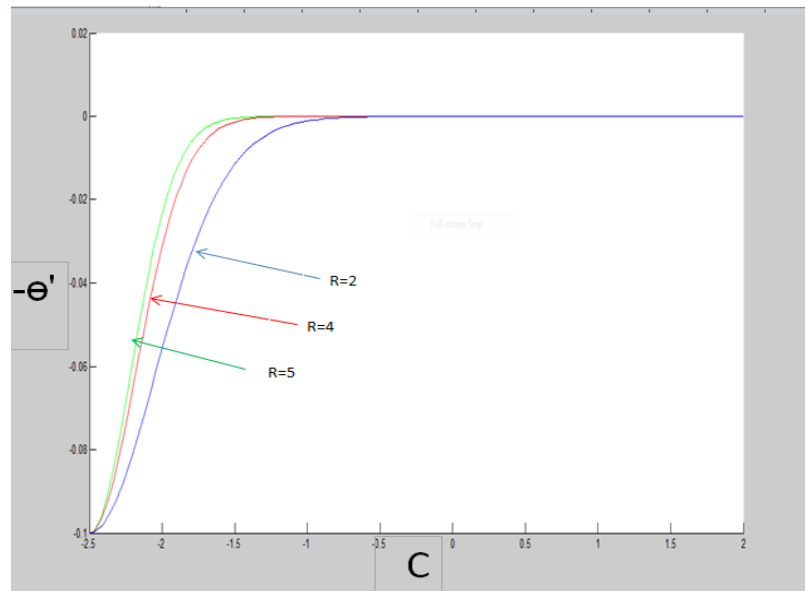

Fig.8. Variation of temperature gradient $-\theta^{\prime}(0)$ with $\mathbf{C}$ for various values of $\mathbf{R}$ when $\beta=0.5, \lambda=1, M=1, K=2$

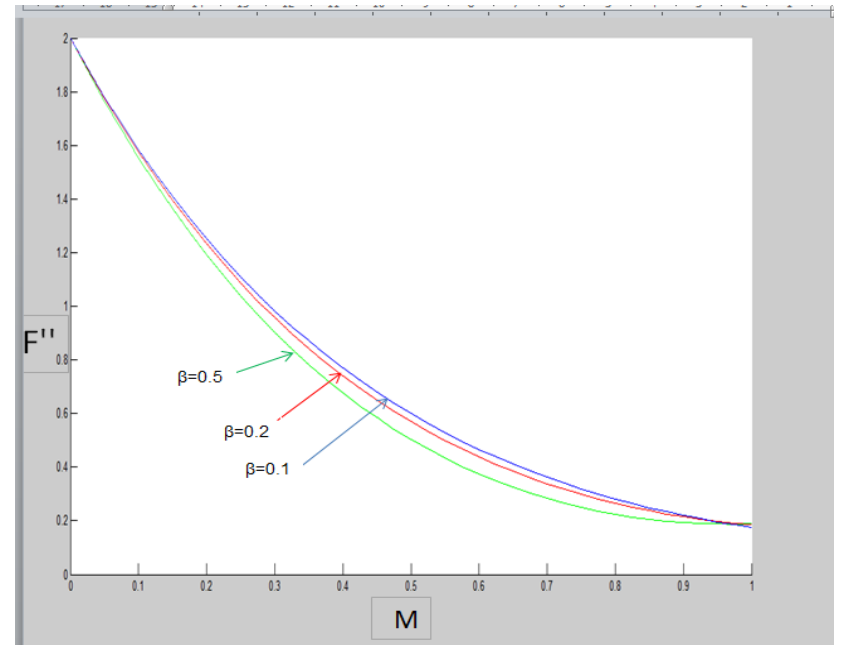

Fig.9. Variation of skin friction coefficient $f^{\prime \prime}(0)$ with $M$ for various values of $\beta$ when $K=2, \lambda=1, R=5$

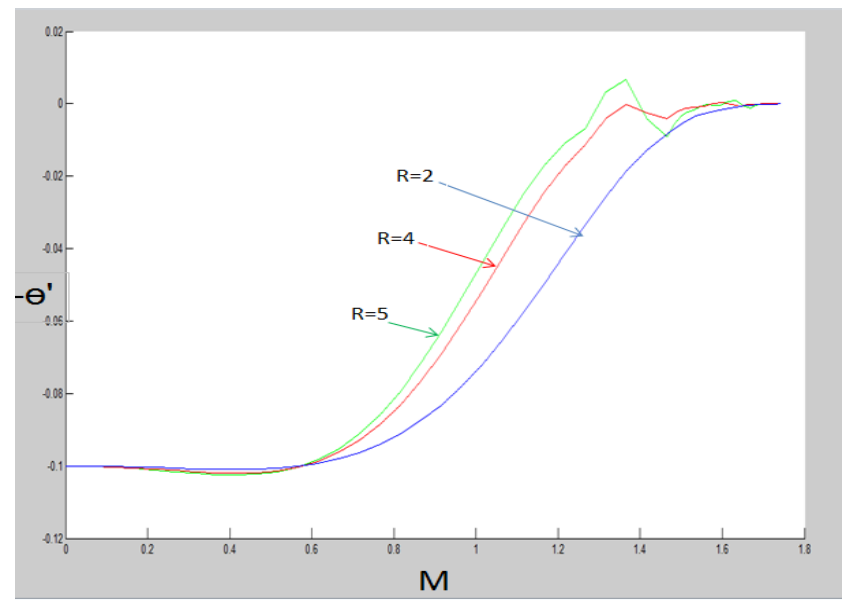

Fig.10. Variation of temperature gradient $-\theta^{\prime}(0)$ with $\mathbf{M}$ for various values of $\mathbf{R}$ when $\beta=0.5, \lambda=1, M=1, K=2$ 


\section{Conclusions:}

The MHD stagnation point flow of Casson fluid and heat transfer over a stretching/ shrinking sheet are investigated taking into consideration the thermal radiation effect. Using similarity transformation the governing equations are transformed to self-similar ODE'S which are then solved using Rang-Kutta method. From the study, the following remarks can be summarized.

1. The velocity is increase when the Casson parameter $\beta$ is increase.

2. The velocity is decrease when the porosity parameter $K$ is increase.

3 . The velocity is decrease when the magnetic field $M$ is increase.

4. The magnitude of wall skin-friction coefficient decreases with Casson parameter $\beta$ is increase.

5. The temperature inside boundary layer decreases when thermal radiation $R$ increases.

\section{$\underline{\text { References }}$}

[1] A. M. Salem and R. Fathy "Effects of variable properties on MHD heat and mass transfer flow near a stagnation point towards a stretching sheet in a porous medium with thermal radiation "Chinese Physics B,Vol.(21), Article ID 054701,(2012).

[2] Homann f, Der Einfluss grosser Zahigkeit bei der stromung um den Zylinder und um die kugel . Zeitschrift fur Angewandte Mathematik and Mechanik,Vol.(16),pp.153-164,(1936).

[3] H. I. Andersson "MHD flow of a viscoelastic fluid past a stretching surface" Acta Mechnica,Vol.(95),no.1-4,p.p.227-230 (1992).

[4] J. Ahmed, A. Shahzad , M. Khan and R. Ali " A note on connective heat transfer of an MHD Jeffrey fluid over a stretching sheet", AIP Advances 5, 117117,(2015); doi : 10.1063/1.4935571.

[5] K. Bhattacharyya "Effects of radiation and heat source/sink on unsteady MHD boundary layer flow and heat transfer over a shrinking sheet with suction/injection "Frontiers of Chemical Engineering, Vol.(5),no.(3),pp.376-384,(2011).

[6] K. Bhattacharyya , "MHD stagnation- point flow of Casson fluid and heat transfer over a stretching sheet with thermal radiation " Journal of Thermodynamics, Article ID 169974,(2013).

[7] K. Bhattacharyya and G. C. Layek "Chemically reactive solute distributive in MHD boundary layer flow over a permeable stretching sheet with suction or blowing "Chemical Engineering Communications, Vol.(197),pp.1527-1540,(2010). 
[8] K. B. Pavlov, "Magneto hydrodynamic flow of an incompressible viscous fluid caused by the deformation of a plane surface", Magneto hydrodynamics, Vol.(10),pp.146-148,(1974).

[9] K. Bhattacharyya, T. Hayat and A. Alsaedi " Exact solution for boundary layer flow of Casson fluid over a permeable stretching / shrinking sheet." Zeitschrift fur Angewandte Mathematik and Mechanik,(2013).

[10] L. J. Crane "Flow past a stretching plate" Zeitschrift fur Angewandte Mathematik and Physik, Vol.(21),no.4,pp.645-647,(1970).

[11] M. Nakamura and T. Sawada ,"Numerical study on the flow of a non-Newtonian fluid through an axisymmetric stenosis ," , Journal of Biomechanical Engineering, Vol.(110), no.2, pp.137-143,(1988).

[12] M. Nandeppanavar and J. M. Shilpa," Stagnation point flow of non -Newtonian fluid and heat transfer over a stretching /shrinking sheet in a porous medium" Chemical and process engineering research, .Vol.(46),(2016).

[13] M. Q. Brewster " Thermal radiative transfer properties " John Wiley and Sons,(1972).

[14] S. Mukhopadhyay, G. C. Layek and S. A. Samad " Study of MHD boundary Layer flow over a heated stretching sheet with variable viscosity" Intr. J. of Heat and Mass Transfer ,Vol. (48),no.21-22,p.p. 4460-4466,(2005).

[15] S. Mukhopadhyay and R. S. R. Gorla "Effects of partial slip on boundary layer Flow past a permeable exponential stretching sheet in a presence of thermal Radiation "Heat and mass transfer,Vol.(48), pp.1773-1781,(2012).

[16] T. Fang and J. Zhang "Closed form exact solutions of MHD viscous flow over a stretching sheet "Communications in Nonlinear Science and Numerical Simulation ,Vol.(197),no.12 , pp.1527-1540,(2010). 